\title{
Heterogeneous Traffic Performance Comparison for 6LoWPAN Enabled Low-Power Transceivers
}

\author{
Mikhail Afanasyev Damien O'Rourke Branislav Kusy Wen Hu \\ CSIRO ICT Centre, Brisbane, Australia \\ \{firstname.lastname\}@csiro.au
}

\begin{abstract}
We study the suitability of IPv6/6LowPan protocols under heterogeneous traffic loads in environmental sensor networks: microclimate nodes generating periodic but low-bandwidth data, while multimedia nodes generating high-bandwidth data streams on demand, for example. We further study the performance tradeoffs we can achieve by varying the radio frequency or data rate of radio transceivers in both indoor and outdoor environments. We use one legacy and two new IEEE 802.15.4 compliant transceivers, in the $900 \mathrm{MHz}$ and $2.4 \mathrm{GHz}$ bands. We show that, surprisingly, the $2.4 \mathrm{GHz}$ radio outperforms the $900 \mathrm{MHz}$ radio outdoors in terms of the maximum range, but performs worse indoors at higher data rates. We show, however, that irrespective of the radio band used, the Berkeley low-power IP network stack supports highbandwidth data transmissions (up to $120 \mathrm{kbps}$ ), while handling heterogeneous traffic well - at $99.9 \%$ reliability.
\end{abstract}

\section{Introduction}

The progress in Wireless Sensor Networks (WSNs) is arguably happening along multiple axes. New IEEE 802.15.4 compliant transceivers provide longer ranges and variable data rates. General communication protocols such as IP [4] provide alternatives to specialised protocols such as the Collection Tree Protocol (CTP) [3]. WSN applications utilise heterogeneous sensors that generate data streams with different data rates, reliability requirements, and latency constraints, in a sharp departure from the homogeneous traffic of Great Duck Island [7] or Golden Gate bridge [6] deployments. It is, therefore, important to understand the design choices we have in developing and deploying WSN applications.

In this paper, we first present a comparative study of two new low-power radio transceivers. Driven partly by the increased interest in WSNs and the "Internet of Things", companies have introduced a number of improvements in transceiver chip designs. To name a few, modern radio chips interoperate through compliance with IEEE

Permission to make digital or hard copies of all or part of this work for personal or classroom use is granted without fee provided that copies are not made or distributed for profit or commercial advantage and that copies bear this notice and the full citation on the first page. To copy otherwise, to republish, to post on servers or to redistribute to lists, requires prior specific permission and/or a fee. HotEMNETS10 June 28-29, 2010, Killarney, Ireland

Copyright 2010 ACM 978-1-4503-0265-4 ...\$5.00
802.15.4, have high reception sensitivity, and provide variable data rates. We perform extensive experimental evaluation of two Atmel radio chips (the AT86RF212 and AT86RF230) and demonstrate the dramatic improvements that these chips offer over a highly popular legacy radio (CC2420). We also quantify the benefits (or lack thereof) of using the $900 \mathrm{MHz}$ frequency band as opposed to the $2.4 \mathrm{GHz}$ one.

The original belief that IP was not suitable for communications in sensor networks, led the research community to design numerous specialised communication protocols such as CTP and Directed Diffusion [5]. Work carried out in [4] shows that IP has been overlooked by the research community but provides extra benefits, such as seamless Internet connectivity with minimum energy overhead. However, to the best of our knowledge, IP has only been shown to be a viable option for sensor networks with homogeneous, low frequency, periodic traffic. We show that, with the latest developments in hardware and software, IP performs well as a general network stack for heterogeneous traffic in sensor networks.

The contributions of this paper are therefore twofold:

- We characterise two new radio transceivers in terms of their maximum communication range, energy consumption, and supported physical data rates and compare them with the most popular currently available platform.

- We analyse the network performance of the transceivers while running IPv6 and evaluate energyefficiency, goodput, and end-to-end packet delivery rates. Furthermore, we demonstrate the feasibility of a general IP-based network stack in sensor networks for heterogeneous traffic which includes both lowvolume periodic (e.g. micro-climate measurements) and high-volume bursty (e.g. multimedia) traffic.

\section{Background and Related Work}

Sensor networks hadn't traditionally been considered with IP in mind as it was considered to be in contradiction to the needs of WSNs. However, as a number of lightweight implementations began to emerge the possibilities of using IP in sensor networks became more apparent. In [1], Dunkels et. al describe their work on a TCP/IP implementation for WSNs and made headway on many of the challenges in this area; later, they also implemented an IPv6 stack for Contiki [2]. In [4], Hui and Culler give a detailed analysis of the performance of IPv6 on a production-class WSN and provide a complete IPv6 based network architecture. 
In this paper we describe experimental results using the Berkeley Internet Protocol (BLIP) stack: an open source implementation of IPv6 and 6LoWPan in TinyOS. BLIP is effectively a 6LoWPan stack with IPv6 header compression, neighbour discovery, default route selection, pointto-point routing, and network programming support. It also provides an implementation of both UDP and TCP, although the former was used exclusively in our experiments due to the experimental state of the latter. Although no standard protocol exists to date for routing in low-power wireless networks the current implementation of BLIP implements a low power routing protocol called HYDRO ${ }^{1}$. However, it is envisaged that BLIP 2.0 will use the Routing Protocol for Low power and lossy networks (RPL) ${ }^{2}$, drafted by the IETF towards a standardisation effort.

Current 6LoWPan implementations have mainly been tested on the Telosb platform, which uses the legacy CC2420 radio. In this work we test BLIP with two new radios (the AT86RF230 and AT86RF212 from Atmel) and present its performance in a number of scenarios.

\section{Characterisation of Radios}

We describe properties of two new radio transceivers, the Atmel RF212 and RF230, and evaluate their performance (along with the CC2420) in field experiments.

\subsection{Transceiver Description}

Table 1 outlines the main features of each radio. The CC2420 is directly comparable to the RF230 in that both operate at a frequency of $2.4 \mathrm{GHz}$ and have a data rate of $250 \mathrm{kbps}$; the RF212, on the other hand, operates at $915 \mathrm{MHz}$ and can select between multiple data rates including the $20 \mathrm{kbps}, 250 \mathrm{kbps}$, and $1 \mathrm{Mbps}$ that we considered in our experiments. As can be seen, the Atmel radios have both a higher sensitivity and maximum transmit power, resulting in improved radio range (cf. Section 3.2).

In order to achieve multiple data rates, the RF212 employs two different modulation schemes and varying length pseudorandom spread-spectrum sequences. The modulation schemes used are BPSK (at $20 \mathrm{kbps}$ ) and OQPSK (at $250 \mathrm{kbps}$ and $1 \mathrm{Mbps}$ ). The reduced length spreading sequence, while allowing for increased data rates, also reduces the sensitivity of the radio, as shown in Table 1.

We also note that the CC2420 only offers a maximum transmission power of $0 \mathrm{dBm}$ whereas the RF230 and RF212 offer up to $3 \mathrm{dBm}$ and $10 \mathrm{dBm}$, respectively. The new transceivers therefore provide designers with increased flexibility in areas such as topology control and energy management.

\subsection{Experimental Setup and Results}

Ideally our radio characterisation would have been carried out in free-space conditions with a path-loss coefficient of exactly 2. As these conditions are difficult to attain in practise it was necessary to use the most benign conditions we could find. We carried out two experiments in two different environments. First, we drove a car along a $1.8 \mathrm{~km}$ straight road which was mostly flat with some hills.

\footnotetext{
${ }^{1}$ http://tools.ietf.org/html/

draft-tavakoli-hydro-01

2 http: //www. ietf.org/id/draft-ietf-roll-rpl-06.
}

Second, we drove a car off-road on a flat grassy area, along multiple directions.

For each experiment, transmission was attempted in one direction only. One group of nodes was stationary, positioned $1.5 \mathrm{~m}$ above the ground. The other group was mounted on top of the car, elevated about $0.4 \mathrm{~m}$ above the roof of the vehicle. Packets were sent at a rate of 10 every second. The receivers collected data such as time-stamp, sequence number, average RSSI, and LQI and a roof-mounted GPS unit collected spatio-temporal data. The vehicle was brought to a halt approximately every $50 \mathrm{~m}$ for about 10 seconds and packets were allowed to accumulate. Data was also recorded while driving at speeds of approximately $5-10 \mathrm{~km} / \mathrm{h}$. In both experiments, we used commonly available antennas of the same physical size that are likely to be used in deployments: $0 \mathrm{dBi}$ gain for the RF212, and $2 \mathrm{dBi}$ for the RF230. We used the built-in PCB antennas for CC2420.

As we noted previously, the propagation characteristics in each experiment were slightly removed from those of ideal free space. At a distance of $400 \mathrm{~m}$, for example, the sizes of the first Fresnel zones are $5.8 \mathrm{~m}$ and $3.5 \mathrm{~m}$ for the $900 \mathrm{MHz}$ and $2.4 \mathrm{GHz}$ bands, respectively. Although these zones would have had an impact on our results this is representative of realistic rural sensor network deployments and is also of much interest.

\subsubsection{Experiment 1}

We drove the car along a straight road, fixed the transmitters $1.5 \mathrm{~m}$ above ground, and mounted the receivers on the roof of the car. We estimated the path-loss coefficients, using RSSI measurements, at 2.1 and 2.5 for the RF230 and RF212 transceiver, respectively. These results confirm the non-ideal conditions of the environment but also show that they were not that far removed.

We summarise the results from experiment 1 in Figures 1(a) and 1(b), produced by averaging the raw data over $10 \mathrm{~m}$ bins. In all experiments we consider the range of each transmitter to be the largest distance at which the PRR is greater than $80 \%$. The first graph shows the range comparison for all radios running at the maximum power. Notice that both the RF230 and RF212 perform approximately the same, despite the fact that the latter had significantly higher transmit power ( $5 \mathrm{~dB}$ more) due to the larger antenna gain. We suspect that the better performance of the $2.4 \mathrm{GHz}$ band is linked to the smaller Fresnel zones at this frequency leading to less interference from ground reflections.

Another interesting feature of the RF212 radios is its ability to transmit data at different data rates. This is a new feature of sensor network radios, thus we plot its effect on the maximum transmission range in Figure 1(b). As noted in Section 3.1, we expect the radio range to decrease with higher data rates due to reduced receiver sensitivity. The results were more dramatic than we expected: the maximum range at $1 \mathrm{Mbps}$ was only $120 \mathrm{~m}$ (vs. $900 \mathrm{~m}$ at $250 \mathrm{kbps}$ ), while communications at $20 \mathrm{kbps}$ were still reliable at the maximum distance we could measure $(1.9 \mathrm{~km})$. The significant difference in radio ranges demonstrates the importance of proper rate selection algorithms.

\subsubsection{Experiment 2}

We conducted another experiment in a different outdoor environment for repeatability. We focused only on 


\begin{tabular}{|c|c|c|c|c|c|c|c|}
\hline & Freq. (MHz) & Data Rate (kbps) & Sens. (dBm) & \multicolumn{2}{|c|}{ Tx } & $\mathrm{Rx}(\mathrm{mA})$ & Max. Pwr (dBm) \\
\hline & & & & $\mathrm{dBm}$ & $\mathrm{mA}$ & & \\
\hline CC2420 & 2400 & 250 & -95 & 0 & 17.4 & 18.8 & 0 \\
\hline RF230 & 2400 & 250 & -101 & 1,3 & $14.5,16.5$ & 15.5 & 3 \\
\hline \multirow{3}{*}{ RF212 } & \multirow{3}{*}{915} & 20 & -110 & \multirow{3}{*}{$0,5,10$} & \multirow{3}{*}{$14,18,24$} & \multirow{3}{*}{9} & \multirow{3}{*}{10} \\
\hline & & 250 & -101 & & & & \\
\hline & & 1000 & -93 & & & & \\
\hline
\end{tabular}

Table 1. Parameters for the new transceivers with the CC2420 listed for comparison.

the RF230 and RF212 operating at their default data rate of $250 \mathrm{kbps}$. We set up four identical receivers for each radio type and we mounted the transmitters on top of the car. We logged the data as before, however, this time we were able to effectively characterise four distinct links at the same time. We drove in a variety of directions, collecting at least eight distinct traces. We assume that any differences in the range caused by interference patterns or reflections would be averaged out by a large number of the distinct measurements. We estimated the path-loss coefficients using the RF230 and RF212 radios to be 2.2 and 2.8 , respectively. As these values are sufficiently close to those obtained in Experiment 1, the two experiments are therefore comparable.

Figure 1(c) shows a summary of the results obtained during the second experiment; we combined the results from all experiments by counting the number of links with a PRR greater than $80 \%$ at each distance from the source. This graphs confirms that the $2.4 \mathrm{GHz}$ band is superior for outdoor communications - for example, at $600 \mathrm{~m}$ none of the RF212 links had good connectivity, while over $40 \%$ of RF230 links could communicate (despite the $5 \mathrm{~dB}$ lower transmitted power of the RF230 transceiver).

\subsubsection{Results Summary}

Table 2 shows the energy consumed by the three radios during transmission and reception using different configurations (e.g. transmission power, data rates, antenna gain and packet size). We measured the power consumption of the radios in the different states, and calculated the energy required to receive and transmit a single 100byte packet (columns $R_{x}$ and $T_{x}$ ). We then calculated the energy consumption using low-power listening, assuming a $500 \mathrm{~ms}$ preamble length and $6 \mathrm{~ms}$ check time. Column Idle $_{p}$ shows the average power consumed while listening, and $T_{x, L P L}$ is the energy required to send an LPL preamble and packet.

In order to properly compare the radios with different transmission ranges, we use the metric $\Gamma_{r}=\frac{\gamma_{p}}{l \times d}$, where $\gamma_{p}$ is the total energy consumed per packet transmission (including that for a reception, but not including LPL idle), $l$ is the payload size in bytes, and $d$ is the transmission range in meters. This metric allows us to quantify the minimum amount of energy that a radio spends in delivering one byte across the channel: calculated using a 100 byte packet cost. Using this metric, Table 2 shows that, at a rate of $250 \mathrm{kbps}$ rate, the new RF230 radio is 3.2 times more efficient when compared with the legacy CC2420 radio.

\section{IPv6 Analysis}

We describe our implementation and results of an eleven-node testbed running IPv6. We used the UC Berkeley 6LoWPAN implementation (BLIP) available in the
TinyOS 2.1 core library. One node was setup as base, running IPBasestationP.nc, providing routes between the PAN and the Internet. The nodes in the PAN were programmed to generate network traffic with varying interpacket intervals and physical data rates; IPv6 statistics were also collected at each node. The nodes of the PAN formed a mesh network using HYDRO (see Section 2) and were monitored using standard tools such as ping6, tracert 6 , and nc6.

56

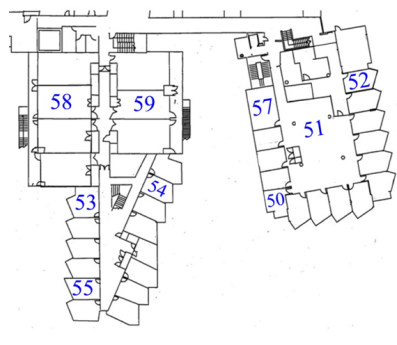

$10 \mathrm{~m}-$

Figure 2. Layout of our test network; the sink node was located in the same room as node 55 .

Figure 2 shows our eleven-node testbed. We programmed the network with two types of traffic patterns: periodic and bursty. In the latter case, each node in turn (chosen in a round-robin fashion) sent between 10 and 150 packets-per-second over a 10 minute interval, while the remaining nodes sent one packet every ten seconds. We analysed the relevant statistics of the network including the end-to-end packet reception rate, goodput, and energy consumption.

\subsection{Implementation Issues}

Even though support for the RF212 radio currently exists in TinyOS 2.1, we had to introduce a few modifications to the source code in order to support our platform and the test scenarios. These included modifying interfaces to change the radio frequency on-the-fly, starting experiments remotely via Remote Procedure Calls (RPCs), implementing reset functionality for our platform, increasing packet size to 100 bytes, and extending statistics collected by BLIP to count the number of parent changes and transmitted beacons.

One of the main problems we encountered during testing was the limited speed of the TinyOS serial driver, which is only one twentieth of the maximum data-rate used (1 Mbps). Therefore, as the aggregate data-rate at the base station increased, some of the incoming packets were dropped at the serial port queue, negatively influencing the reliability metrics. We solved this problem by compressing the data sent to the PC down to four bytes (node id and 


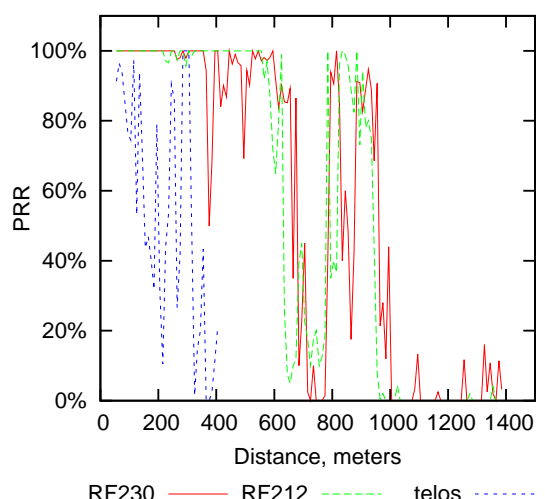

(a) $250 \mathrm{kbps}$, all radios

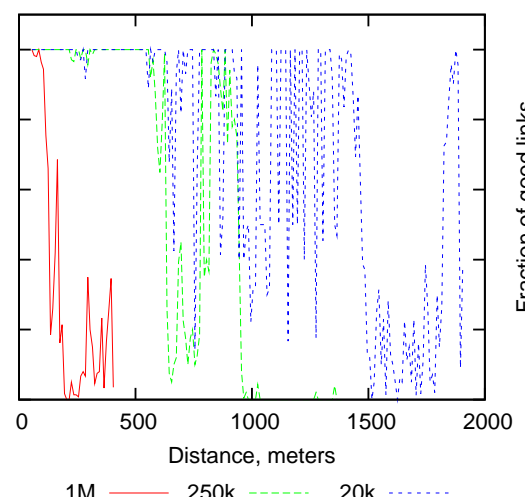

(b) RF212 only, 3 rates

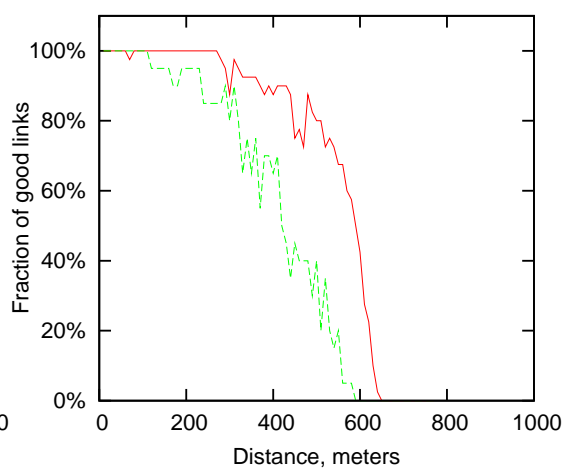

RF230 - RF212

(c) $250 \mathrm{kbps}$, multiple links

Figure 1. Single trial results from experiment 1: 1(a), and 1(b) along with combined results for experiment 2, 1(c).

\begin{tabular}{|c|c|c|c|c|c|c|c|c|c|}
\hline & \multirow{2}{*}{$\begin{array}{c}\text { Pwr } \\
(\mathrm{dBm})\end{array}$} & \multirow{2}{*}{$\begin{array}{l}\text { Rate } \\
\text { (kbps) }\end{array}$} & \multirow{2}{*}{$\begin{array}{c}\text { Antenna } \\
\text { Gain }(\mathrm{dBi})\end{array}$} & \multicolumn{2}{|c|}{ No LPL } & \multicolumn{2}{|c|}{ LPL } & \multirow{2}{*}{ Range (m) } & \multirow{2}{*}{$\Gamma_{r}\left(\mathrm{nJm}^{-1}\right)$} \\
\hline & & & & $R_{x}(m \mathrm{~J})$ & $T_{x}(m \mathrm{~J})$ & $T_{x, L P L}(m \mathbf{J})$ & $I d l e_{p}(m \mathrm{~W})$ & & \\
\hline RF230 & 3 & 250 & 2 & 0.23 & 0.30 & 37.5 & 0.73 & 850 & 443 \\
\hline \multirow{3}{*}{ RF212 } & \multirow{3}{*}{10} & 20 & \multirow{3}{*}{0} & 2.0 & 3.79 & 32.25 & 0.17 & 1,850 & 185 \\
\hline & & 250 & & 0.16 & 0.35 & 28.81 & 0.17 & 920 & 314 \\
\hline & & $1 \mathrm{M}$ & & 0.06 & 0.15 & 29.06 & 0.18 & 100 & 2911 \\
\hline CC2420 & 0 & 250 & 0 & 0.26 & 0.72 & 32.19 & 0.25 & 320 & 1014 \\
\hline
\end{tabular}

Table 2. Results of range test experiments for each setup. The energy calculations assume a voltage of $3.0 \mathrm{~V}$ is used.

sequence number) if high data load was detected.

We instrumented the radio stack with GPIO pins acting as triggers during different stages of packet transmission and reception (i.e. idle, CSMA, packet reception, and packet transmission). We used an oscilloscope to characterise the power consumption of the different stages. The memory footprint of our test program is listed in Table 3 .

\begin{tabular}{|c|c|c|}
\hline & IPBasetation & Application \\
\hline RAM & 7,423 & 6,473 \\
\hline ROM & 23,474 & 39,420 \\
\hline
\end{tabular}

Table 3. Memory footprint of BLIP in bytes (packet size is 102 bytes).

\subsection{Results}

We now discuss the results obtained during the experiments just outlined. We first consider the periodic traffic pattern and then the bursty pattern.

\subsubsection{Periodic traffic pattern}

In this setup we emulated a network collecting lowrate traffic: every node in the network was programmed to transmit a 90 -byte packet every 10 seconds, in one case, and every 2 minutes, in another. In each case the experiment was run over a 10 -hour period. We repeated this for both the RF212 and the RF230, the former being run at the most popular $250 \mathrm{kbps}$.

Table 4 shows our experimental results. In all configurations, the networks achieved a packet reception ratio of $99.9 \%$ or better. We calculated the route change interval as the average time between change of parent node for each station. In order to estimate the amount of overhead generated by the network, we calculated the fraction of network management (ICMP) packets over the total sent and received by the each node. These were mostly route advertisements, with a smaller fraction $(9 \%$ to $27 \%$, depending on the experiment) being route solicitations. Finally, we calculated the average energy required to transmit one packet; this included the energy to forward the packet and that for management packets.

\subsubsection{Bursty traffic pattern}

In the next experiment we emulated a multimedia sensor network in which one node transmitted data at a fast rate (100's of packets per second). We used UDP in order to enable high-rate packet transmission. Currently, the TCP implementation in BLIP is still in its experimental stages $^{3}$ and we therefore avoided using it. No flow or congestion control algorithms were employed; instead, the network sent packets at a fixed rate.

We tested three configurations: the RF212 radio running at $250 \mathrm{kbps}$, at $1 \mathrm{Mbps}$, and the RF230 radio running at $250 \mathrm{kbps}$. We used four different nodes located at varying distances from the gateway as transmitters operating over 16 different transmission rates in the range of 20300 packets-per-second. Each transmitter sent packets at a fixed rate for approximately 10 minutes while the reception rate was measured at the gateway.

Figures 3(a)-3(c) show the results of the experiments. For the $250 \mathrm{kbps}$ rate, both the RF212 and the RF230 achieve about $120 \mathrm{kbps}$ over one-hop links - about $48 \%$

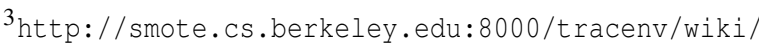
blip
} 


\begin{tabular}{|c|c|c|c|c|}
\hline \multirow{2}{*}{ Property } & \multicolumn{2}{|c|}{ RF212 } & \multicolumn{2}{c|}{ RF230 } \\
\cline { 2 - 5 } & $10 \mathrm{sec} / \mathrm{pkt}$ & $120 \mathrm{sec} / \mathrm{pkt}$ & $10 \mathrm{sec} / \mathrm{pkt}$ & $120 \mathrm{sec} / \mathrm{pkt}$ \\
\hline End-to-end PRR & $100.0 \%$ & $100.0 \%$ & $99.9 \%$ & $99.9 \%$ \\
Average depth (hops) & 1.56 & 1.89 & 1.96 & 1.89 \\
Average route change interval & $104 \mathrm{~min}$ & $36 \mathrm{~min}$ & $75 \mathrm{~min}$ & $333 \mathrm{~min}$ \\
\hline Fraction of ICMP in received packets & $2.54 \%$ & $9.91 \%$ & $0.41 \%$ & $1.41 \%$ \\
Fraction of ICMP in sent packets & $32.0 \%$ & $44.7 \%$ & $4.44 \%$ & $16.7 \%$ \\
\hline Total energy per data packet (mJ) & 97.9 & 90.7 & 80.7 & 159.8 \\
Station energy per hour (J) & 1.72 & 0.27 & 2.9 & 0.49 \\
\hline
\end{tabular}

Table 4. Periodic traffic characterisation.

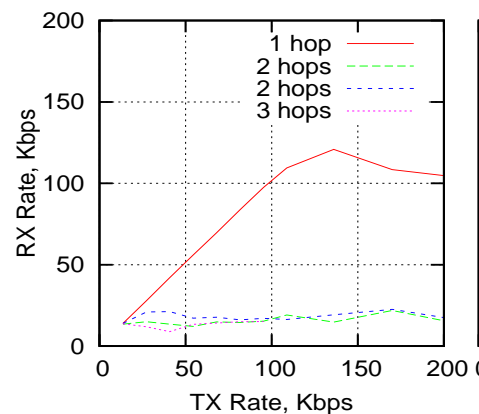

(a) RF230, $250 \mathrm{kbps}$

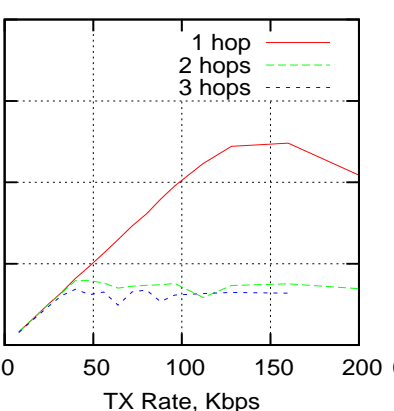

(b) RF212, $250 \mathrm{kbps}$

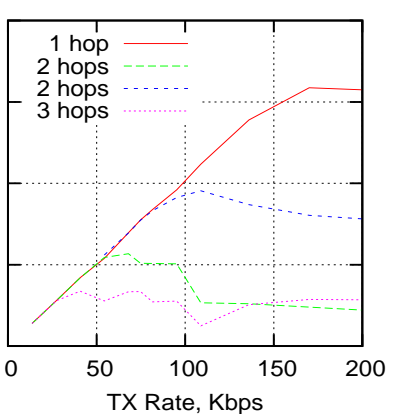

(c) RF212, $1 \mathrm{Mbps}$

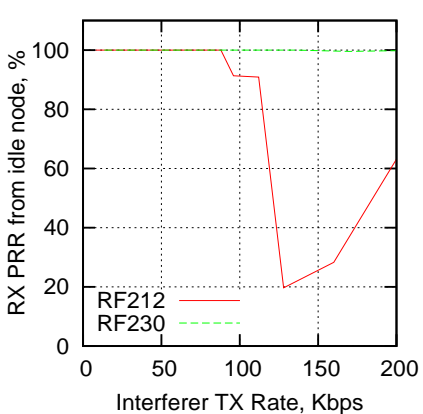

(d) Interference test

Figure 3. Results obtained for different IPv6 configurations.

of their capacity. With multiple hops, the performance decreases further in both cases. However, this decrease is more pronounced for the RF230 which we suspect could be caused either by local $2.4 \mathrm{GHz}$ interference or by immature TinyOS driver implementation. For a data rate of 1 Mbps on the RF212 we note that the one-hop throughput increases to approximately $160 \mathrm{kbps}$. In this case the back-off and preamble rate remains at $250 \mathrm{kbps}$ limiting the effects of the data-rate increase. However, we can still see the main benefits of the higher data rates over multiple hops: one of the 2-hop links shows a performance improvement by more than a factor of three, for example.

In order to evaluate the performance of the heterogeneous setup, we measured the effects of the high-rate nodes on the low-rate nodes. We set up a "victim" node 2-hops away from the gateway, to transmit a packet every 10 seconds. Then, an "interfering" node, which was one hop away from the gateway, began to transmit packets at a high rate. We measured the PRR at the victim node during this time. The experiment was performed using both the RF212 and RF230 networks running at $250 \mathrm{Kbps}$. Figure 3(d) shows the results: there is no packet loss from the victim node provided the transmission rate of the interferer is less than $70 \mathrm{kbps}$. This shows that the system is suitable for mixed-traffic applications.

\section{Conclusions and Future Work}

We have evaluated and analysed the performance of IPv6 and two new low-power sensor network radios over a single link and an eleven-node testbed running TinyOS and 6LoWPan extensively. Our results show that the latest developments in hardware and software make general IPbased network stacks feasible for heterogeneous traffic in sensor networks. Future work will include studies on congestion control for networks of this type; TCP is too com- plex for low-power wireless networks and does not work well on lossy links by making the assumption that packet losses are the result of network congestion only.

\section{References}

[1] A. Dunkels, T. Voigt, and J. Alonso. Making TCP/IP viable for wireless sensor networks. In $E W S N^{\prime} 04$, Jan 2004.

[2] M. Durvy, J. Abeillé, P. Wetterwald, C. O’Flynn, B. Leverett, E. Gnoske, M. Vidales, G. Mulligan, N. Tsiftes, N. Finne, and A. Dunkels. Making sensor networks IPv6 ready. In SenSys '08, pages 421-422, New York, NY, USA, 2008. ACM.

[3] O. Gnawali, R. Fonseca, K. Jamieson, D. Moss, and P. Levis. Collection tree protocol. In SenSys '09, pages 1-14, New York, NY, USA, 2009. ACM.

[4] J. W. Hui and D. E. Culler. Ip is dead, long live ip for wireless sensor networks. In SenSys '08, pages 15-28, New York, NY, USA, 2008. ACM.

[5] C. Intanagonwiwat, R. Govindan, D. Estrin, J. Heidemann, and F. Silva. Directed diffusion for wireless sensor networking. IEEE/ACM Trans. Netw., 11(1):216, 2003.

[6] S. Kim, S. Pakzad, D. Culler, J. Demmel, G. Fenves, S. Glaser, and M. Turon. Health monitoring of civil infrastructures using wireless sensor networks. In IPSN '07, pages 254-263, New York, NY, USA, 2007. ACM.

[7] R. Szewczyk, E. Osterweil, J. Polastre, M. Hamilton, A. Mainwaring, and D. Estrin. Habitat monitoring with sensor networks. Commun. ACM, 47(6):34-40, 2004. 